\title{
On the Asymptotic Stability of Some Particular Differential Equations
}

\author{
Necdet Bildik*, Sinan Deniz \\ Celal Bayar University, Faculty of Arts and Science, Department of Mathematics, Muradiye Campus, 45030, \\ Manisa, Turkey. \\ * Corresponding author. Tel.: +(90)0236 201 3203; email: necdet.bildik@cbu.edu.tr \\ Manuscript submitted March 31, 2015; accepted October 12, 2015. \\ doi: 10.17706/ijapm.2015.5.4.252-258
}

\begin{abstract}
The importance of differential equations in scientific world is a crystal-clear fact. Many problems in physics, ecology, biology, engineering etc. can be modeled by ordinary differential equations and very few of them can be solved in terms of elementary functions. However, it is possible to reveal the main features of the solutions by the help of qualitative methods. In this study, we try to show that the conditions for some nonlinear equations to be asymptotically stable.
\end{abstract}

Key words: Liapunov's second method, ordinary differential equations, stability analysis.

\section{Introduction}

"Stability" is a very important concept for nonlinear analysis. For instance, roughly speaking, a physical system is called stable if small changes at some times causes only a small change in the behavior of the system in the future. When analytical or quantitative methods remain incapable to solve the differential equations of modeled problems, we use qualitative methods to understand the behavior of the solutions. These methods are very useful because it depends only on the nature of the equations. In these methods, we do not need the solution itself, but the stability of the solution. There are many studies about "stability" in the literature [1]-[5].

Liapunov's methods and Liapunov functions have great importance in the stability theory and applications [6]-[9]. Because, the existence of Liapunov functions is a necessary and sufficient condition to determine the stability of many differential equations. Besides, we do not need to know any knowledge of the solution of differential equations. There is no general technique for constructing Liapunov functions. However, in many specific cases, the construction of Liapunov functions is known. This method can be taken as a generalization of two physical principles for conservative systems. If the potential energy is a local minimum, we say a rest position is stable, otherwise it is unstable, and the total energy is a constant during any motion.

In this paper, we use Liapunov's second method to define the behavior of solution of some nonlinear differential equations. Especially, we are looking for the asymptotic stability of the solutions. Asymptotic stability requires two things. The solution be Liapunov stable and in the phase plane all half-paths starting sufficiently near to the origin at a time $t_{0}$ approach the origin. For further informations about Liapunov methods, seeing [10], [11]. 


\section{Definitions and Theorems}

In this section we give some definitions and theorems about asymptotic stability in order to reach our main results. For further information, theorems and proofs we refer to [10], [11].

Definition 1: Let $N$ be the any simple 'patch' on the $x, y$ plane covering the origin with its boundary is excluded. Then it is called as connected neighbourhood of the origin.

Definition 2: Let $V(x, y), \frac{\partial V}{\partial x}, \frac{\partial V}{\partial y}$ are continuous and $V(x, y)>0$ except the origin in some connected neighbourhood $N$ of the origin. If there exists a number $\xi$ such that, for every value of the parameter $\alpha$ in the interval $0<\alpha<\xi$, the equation $V(x, y)=\alpha$ for $(x, y)$ in $N$ defines, uniquely, a simple closed curve $T_{\alpha}$ in $N$ which surrounds the origin. Then the family of curves $V(x, y)=\alpha$, $0<\alpha<\xi$, is called a topographic system on $N_{\xi}$, where $N_{\xi}$ is a connected neighbourhood of the origin defined by $V(x, y)<\xi$, where $N_{\xi} \subseteq N$.

Theorem 1: Let $V(x, y)$ satisfy the conditions of a topographic system in $N_{\xi}$ and let the system $\dot{x}=X(x, y), \dot{y}=Y(x, y)$ be regular in $N_{\xi}$.Suppose also that $\dot{V}(x, y)<0$ in the region $R_{\xi}$, defined to be the neighbourhood $N_{\xi}$ with the origin excluded. Then there are no equilibrium points in $R_{\xi}$ except the origin and $N_{\xi}$ does not contain any limit cycles.

Theorem 2: Let $V(x, y)$ satisfy the conditions of a topographic system, and in the neighbourhood $N_{\xi}$ of the definition let the system $\dot{x}=X(x, y), \dot{y}=Y(x, y)$ be regular and have an equilibrium point at the origin. Suppose also that $\dot{V}(x, y)<0 \quad$ in the region consisting of $N_{\xi}$ with the origin excluded. Then the zero solution is uniformly and asymptotically stable.

One of the important things in this area is to find the domain of asymptotic stability of the origin. If we have a neighbourhood $N_{\xi}$ from which all half-paths approach the origin as $t \rightarrow \infty$, then this neighbourhood is called a domain of asymptotic stability or a domain of attraction for the origin. Generally, it is not the largest possible domain of asymptotic stability, since it depends on the Liapunov function chosen. If the domain of asymptotic stability consists of the whole $x, y$ plane, the system is said to be globally asymptotically stable.

\section{Stability of Lienard Type Equations}

In this section, we use some particular equations to examine theirs stability properties. These equations are the type of Lienard equation [12]-[14]. This equation is a second order differential equation which were studied during the development of radio and vacuum tube technology. Oscillating circuits can be also modeled by these equations. If $f$ is an even function, $g$ is an odd function and they are continuously differentiable on $R$, Lienard equation is defined as

$$
\frac{d^{2} x}{d t^{2}}+f(x) \frac{d x}{d t}+g(x)=0
$$

In order to use Liapunov functions, we convert this equation to a two dimensional system 


$$
\left[\begin{array}{l}
\dot{x} \\
\dot{y}
\end{array}\right]=\left[\begin{array}{l}
y-F(x) \\
-g(x)
\end{array}\right]
$$

where

$$
F(x)=\int_{0}^{x} f(\xi) d \xi
$$

Now, let us investigate the asymptotic stability for Lienard type equations. In order to guarantee the asymptotic stability, we need to give some conditions on the functions $f(x)$ and $g(x)$. We first let

$$
G(x)=\int_{0}^{x} g(\xi) d \xi
$$

and we suppose that $g(x)$ is positive/negative when $x$ is positive/negative for all $x$.Therefore $G(x)>0$ for all $x \neq 0$. We now need to guess a Liapunov function which will be positive definite for all $x$. Let us consider the function $V(x, y)=G(x)+\frac{1}{2} y^{2}$ which yields that

$$
\begin{aligned}
\dot{V}(x, y) & =\frac{\partial V}{\partial x} \dot{x}+\frac{\partial V}{\partial y} \dot{y}=g(x) \dot{x}+y \dot{y} \\
& =g(x)(y-F(x))+y(-g(x))=-g(x) F(x) .
\end{aligned}
$$

If $f(x)>0$ for all $x$, then the last equation will be negative definite for all $x$. Thus all solutions from all initial positions eventually go to the origin. That is, the system is globally asymptotically stable.

$$
\text { 4. Stability of the Equations Type of } \begin{array}{r}
\dot{x}=a(x)+\beta y \\
\dot{y}=b(x)+\delta y
\end{array}
$$

In this part, we investigate the suitable Liapunov function to determine the domain of asymptotic stability of the origin for the given system. As known, there is no general formula for the Liapunov functions. We need to offer some functions as a candidate Liapunov polynomials.

One of the most encountered type of the equations in qualitative theory is $\begin{gathered}\dot{x}=a(x)+\beta y \\ \dot{y}=b(x)+\delta y\end{gathered}$. These systems may be the model of some substantial applications. So it is important to search for their stability properties.

If we have the conditions

$$
[\delta a(x)-\beta b(x)] x>0, x a(x)+\delta x^{2}<0, x \neq 0
$$

in some neighbourhood of the origin, then we can take the function

$$
V(x, y)=(\delta x-\beta y)^{2}+2 \int_{0}^{x}[\delta a(\xi)-\beta b(\xi)] d \xi
$$

as a strong Liapunov function for the zero solution of the system 


$$
\begin{aligned}
& \dot{x}=a(x)+\beta y \\
& \dot{y}=b(x)+\delta y
\end{aligned}
$$

where

$$
f(0)=0, g(0)=0
$$

The proof is very easy. Firstly, it is ready to see that the function in (7) is positive definite if

$$
[\delta a(x)-\beta b(x)] x>0 .
$$

Taking the derivative of this function gives

$$
\dot{V}(x, y)=2[\delta a(x)-\beta b(x)][\delta x+a(x)] .
$$

The last equation is negative definite if and only if

$$
[\delta a(x)-\beta b(x)][\delta x+a(x)]<0
$$

in a deleted neighbourhood of the origin, namely $x \neq 0$. Inequalities (10) and (12) yields

$$
x a(x)+\delta x^{2}<0
$$

where $x \neq 0$.

\section{Examples}

Example 1) Consider the Lienard equation $\ddot{x}+x^{4} \dot{x}+x^{5}=0$.

Solution: Here we have $f(x)=x^{4}$ and $g(x)=x^{5}$. They are chosen since the conditions in section 3 are satisfied. Then we get

$$
F(x)=\int_{0}^{x} u^{4} d u=\frac{1}{5} x^{5} \quad \text { and } \quad G(x)=\int_{0}^{x} u^{3} d u=\frac{1}{4} x^{4}
$$

This equation can also be written as:

$$
\left[\begin{array}{c}
\dot{x} \\
\dot{y}
\end{array}\right]=\left[\begin{array}{c}
y-\frac{1}{5} x^{5} \\
-x^{5}
\end{array}\right] .
$$

We proceed as explained in Section 3 to obtain the Liapunov function as

$$
V(x, y)=G(x)+\frac{1}{2} y^{2}=\frac{1}{6} x^{6}+\frac{1}{2} y^{2} .
$$

So, 


$$
\dot{V}(x, y)=-g(x) F(x)=-\left(x^{5}\right)\left(\frac{1}{5} x^{5}\right)=-\frac{1}{5}<0 .
$$

This means that zero solution is globally asymptotically stable. Hence solutions from all initial positions ultimately approach the origin.

Example 2) Consider the equation $\ddot{x}+\varepsilon\left(x^{4}-1\right) \dot{x}+x=0$ for $\varepsilon<0$.

Solution: Firstly, we write the equation as a system:

$$
\left[\begin{array}{c}
\dot{x} \\
\dot{y}
\end{array}\right]=\left[\begin{array}{c}
y-\varepsilon\left(\frac{1}{5} x^{5}-x\right) \\
-x
\end{array}\right] .
$$

Now, we need to guess a Liapunov function which needs to be positive definite. Consider again the function that we mentioned before:

$$
V(x, y)=G(x)+\frac{1}{2} y^{2}=\frac{1}{2} x^{2}+\frac{1}{2} y^{2}
$$

So

$$
\dot{V}(x, y)=-g(x) F(x)=-(x)\left(\varepsilon\left(\frac{1}{5} x^{5}-x\right)\right)=\varepsilon\left(x^{2}-\frac{1}{5} x^{6}\right) .
$$

Last equation is negative for $|x|<\sqrt[4]{5}$ and $x \neq 0$. Under these conditions we say that $V(x, y)$ is a Liapunov function for this equation and the zero solution is asymptotically stable. In order to determine the domain of the asymptotic stability, we need to find the largest topographic curve of the Liapunov function. We know that $x^{2}<\sqrt{5}$. Therefore we can select the circle $x^{2}+y^{2}<\sqrt{5}$ as a simple closed curve that is suitable for our conditions and the definition of the topographic curve. In other words, all solutions starting from initial conditions inside the circle $x^{2}+y^{2}<\sqrt{5}$ will tend to the origin.

Example 3) Consider the system

\section{Solution:}

$$
\begin{aligned}
& \dot{x}=-x^{5}+2 x^{6}+y \\
& \dot{y}=-x^{6}-y
\end{aligned} .
$$

For our particular system, we have $a(x)=-x^{5}+2 x^{6}, b(x)=-x^{6}, \beta=1$ and $\delta=-1$ according to the Section 4.

Firstly, we see that

$$
[\delta a(x)-\beta b(x)] x=\left[x^{5}-2 x^{6}+x^{6}\right] x=x^{6}-x^{7}=x^{6}(1-x)>0
$$

for $0<|x|<1$ and

$$
x a(x)+\delta x^{2}=-x^{6}+2 x^{7}-x^{2}<0
$$


in some deleted interval about the origin. Since

$$
2 x^{7}-x^{6}-x^{2}=x^{2}(x-1)\left(2 x^{4}+x^{3}+x^{2}+x+1\right)
$$

has only zeros at $x=0$ and $x=1$, we can be more specific and say that $\dot{V}$ is negative definite also in $0<|x|<1$. Therefore the zero solution is asymptotically stable.

As a result, the Liapunov function is

$$
\begin{aligned}
& V(x, y)=(-x-y)^{2}+2 \int_{0}^{x}\left[-\left(-\xi^{5}+2 \xi^{6}\right)-\left(-\xi^{6}\right)\right] d u \\
& =(x+y)^{2}+2 x^{6}\left[\frac{1}{6}-\frac{x}{7}\right]
\end{aligned}
$$

A domain of asymptotic stability will be the interior of the largest level curve

$$
(x+y)^{2}+2 x^{5}\left[\frac{1}{5}-\frac{x}{6}\right]=C .
$$

which is within $|x|<1$ and closed curve.

\section{Conclusion}

In this study, we use some theorems to determine the asymptotic stability of certain equations. We also try to find the domain of asymptotic stability of these equations which is very important since we can deduce a domain of initial conditions from which the solutions tend to the origin. These qualitative techniques are very useful to understand the behavior of solutions of differential equations that we cannot solve analytically.

\section{References}

[1] Griffiths, D. V., \& Lane, P. A. (1999). Slope stability analysis by finite elements. Geotechnique, 49(3), 387-403.

[2] Morgenstern, N. R., \& Price, V. E. (1965). The analysis of the stability of general slip surfaces. Geotechnique, 15(1), 79-93.

[3] Daafouz, J., Pierre, R., \& Claude, I. (2002). Stability analysis and control synthesis for switched systems: a switched Lyapunov function approach. IEEE Transactions on Automatic Control, 47(11), 1883-1887.

[4] Seydel, R. (2010). Practical Bifurcation and Stability Analysis. New York: Springer.

[5] Hughes, T. J. R., \& Liu, W. K. (1978). Implicit-explicit finite elements in transient analysis: Stability theory. Journal of Applied Mechanics, 45(2), 371-374.

[6] Johansson, M., \& Anders, R. (1998). Computation of piecewise quadratic Lyapunov functions for hybrid systems. IEEE Transactions on Automatic Control, 43(4), 555-559.

[7] Coron, J.-M., Brigitte, d'Andrea-N., \& Georges, B. (2007). A strict Lyapunov function for boundary control of hyperbolic systems of conservation laws. IEEE Transactions on Automatic Control, 52(1), 2-11.

[8] Mori, Y., Takehiro, M., \& Yasuaki, K. (1997). A solution to the common Lyapunov function problem for 
continuous-time systems. Proceedings of the 36th IEEE Conference on Decision and Control, vol. 4.

[9] Clarke, F. H., Yu, S. L., \& Ronald, J. S. (1998). Asymptotic stability and smooth Lyapunov functions. Journal of differential Equations, 149(1), 69-114.

[10] La, S., Joseph, P., \& Solomon, Lefschetz. (1961). Stability by Liapunov's Direct Method: With Applications. New York: Academic Press.

[11] Jordan, D. W., \& Peter, S. (2007). Nonlinear Ordinary Differential Equations: An Introduction for Scientists and Engineers. New York.

[12] Sansone, G., \& Roberto, C. (1964). Non-linear Differential Equations. Oxford: Pergamon Press.

[13] Burton, T. A. (1965). The generalized Liénard equation. Journal of the Society for Industrial and Applied Mathematics, Series A: Control, 3(2), 223-230.

[14] F. S., Zhao. (2002). On explicit exact solutions for the Lienard equation and its applications. Physics Letters A, 293(1), 50-56.

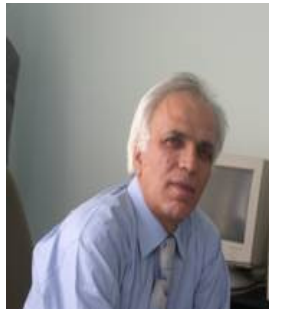

Necdet Bildik was born in Sivas, Turkey in 1951. He graduated from Ankara University in 1974. He earned the M.Sc. degree from University of Louisville, Kentucky, USA in 1978. He awarded the Ph.D. degree in Oklahoma State University, USA in 1982. He was assistant professor in 1988 and also he was became associate professor in 1995. He was promoted to be professor in 2003. He is interested in numerical analysis, ordinary, partial and non-linear differential equations, ergodic theory, stability theory. He has over than a hundred published articles in the national and international journals and conferences. He also serves as a reviewer for many international journals.

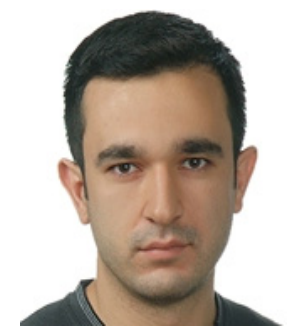

Sinan Deniz was born in Uşak, Turkey in 1989. He is research assistant at the Department of Mathematics in Celal Bayar University. He obtained his B.Sc degree from the Department of Mathematics at Fatih University, İstanbul, Turkey in 2012 and he earned his M.Sc. degree from the Department of Mathematics at Celal Bayar University, Manisa, Turkey. He is also studying for Ph.D degree at Celal Bayar Universtiy. He works on the systems of delay differential equations and numerical solutions of nonlinear differential equations, analytical methods for partial differential equations. 